\title{
THE HUMAN MILK MICROBIOME AND FACTORS INFLUENCING ITS COMPOSITION AND ACTIVITY
}

Carlos Gomez-Gallego, Ph. D. (cargom@utu.fi) ${ }^{1}$; Izaskun Garcia-Mantrana, Ph. D.

(igama@iata.csic.es) ${ }^{2}$, Seppo Salminen, Prof. Ph. D. (sepsal@utu.fi) ${ }^{1}$, María Carmen

1. Functional Foods Forum, Faculty of Medicine, University of Turku, Itäinen Pitkäkatu 4 A, 20014, Turku, Finland. Phone: +358 23336821.

2. Institute of Agrochemistry and Food Technology, National Research Council (IATACSIC), Department of Biotechnology. Valencia, Spain. Phone: +34 963900022

*To whom correspondence should be addressed.

-IATA-CSIC, Av. Agustin Escardino 7, 49860, Paterna, Valencia, Spain. Tel. +34 963900022;

E-mail: $\underline{\text { mcolam@iata.csic.es }}$

8

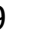




\section{SUMMARY}

2 Beyond its nutritional aspects, human milk contains several bioactive compounds, such as

3 microbes, oligosaccharides, and other substances, which are involved in host-microbe

4 interactions and have a key role in infant health. New techniques have increased our

5 understanding of milk microbiota composition, but little data on the activity of bioactive

6 compounds and their biological role in infants is available. While the human milk microbiome

7 may be influenced by specific factors, including genetics, maternal health and nutrition, mode of

8 delivery, breastfeeding, lactation stage, and geographic location, the impact of these factors on

9 the infant microbiome is not yet known. This article gives an overview of milk microbiota

10 composition and activity, including factors influencing microbial composition and their

11 potential biological relevance on infants' future health.

14 Keywords: milk microbiota, lactation, C-section, diet, gestational age 
1

2 Recent reports have revealed the importance of our gut microbiome for optimal health.

3 Accumulating evidence highlights the potential role of microbes in metabolic, immunological,

4 and microbial programming [1]. Microbes are among the most important environmental factors

5 providing specific signals to guide immune system development and maturation [2, 3]. Shifts in

6 microbiota composition and activity appear to be related to adverse human health outcomes [4].

7 The maternal microbial environment impacts the newborn's immune development and,

8 consequently, the infant's health both early and later on in life. The maternal microbiota is now

9 recognized as a significant determinant of the maternally transferred factors that impact the child's health $[5,6]$. Outcomes can be affected by specific perinatal factors that also alter infant microbiome development. For example, excessive use of antibiotics, unbalanced diet, increasing incidence of caesarean section (C-section) deliveries, unnecessarily stringent hygiene, and continuous stress influence the maternal microbiome. Alterations and disturbances in microbiota composition along with a reduction in microbial diversity or richness have been described as strong risk factors for the development of lifestyle diseases, such as allergies, diabetes, obesity, and metabolic syndrome, irritable bowel syndrome and other inflammatory-related problems $[4$, 7]. The maternal microbiota and infant diet play a key role in infant growth, adequate microbial colonization, immune system maturation, and metabolic development. Then, the infant stepwise microbial colonization process will have an impact on metabolic and immunological response, and these in turn may have an impact on programming of health later in life $[3,8]$. This review aims to describe the impact of the maternal microbiota on infant health through breastfeeding. The review will also provide a broad overview of milk microbiota composition and activity, along with the factors influencing microbial composition and their potential biological relevance.

\section{Human breast milk: More than infant food}

Human milk (HM) provides complete nutrition for the infant. HM's energy, nutrients, and bioactive components directly influence the development of newborn infants. Among its 
bioactive factors, HM contains several immune substances, such as immunoglobulins, cytokines and chemokines, growth factors, hormones, and antibodies. It also contains other nonspecific compounds, such as specific peptides, lactoferrin and other whey proteins, oligosaccharides and a large number of bacteria [9]. All these components are transferred to the infant through breastfeeding.

$\mathrm{HM}$ is the most relevant postnatal element for the metabolic and immunological programming of the infant's health $[10,11]$. Breastfeeding shapes the infant immune system development and also, it is needed for adequate gut function and immune homeostasis maintenance [12]. Breastfed infants have been shown to have a reduced risk of necrotizing enterocolitis and diarrhea, allergy and asthma, inflammatory bowel disease, diabetes, and obesity, among other problems [13]. In addition, differences in gut microbiota composition and activity between exclusively breast-fed and formula-fed infants have been widely reported [14-18]. Breast milk is also a postnatal microbial link as its high diversity of microbes drives the infant's microbial colonization [19-27]. It has been reported that specific maternal gut microbial strains belonging to Bifidobacterium and Staphylococcus spp. are transmitted to infants [28-31]. These data suggest a unique link between family members as specific strains are present in each motherinfant pair. The findings also suggest that transfer of aberrant microbiota from the mother is possible. Altogether, it is important to define the milk microbiota composition and factors that may be transferrable as commensal bacteria in human milk. For example, transmission of lactic acid bacteria and Bifidobacteria from the breastfeeding mother may form a natural protective mechanism that improves development of the infant's gut microbiota for later resilience and reduction in the risk of diarrheal and other dysbiosis-related problems. Improved knowledge in this area may suggest novel means of modulating the maternal and infant microbiota in order to potentially reduce the risk of specific microbiota-associated diseases. (Figure 1)

\section{Human breast milk microbiome}

Historically, human breast milk has been considered an almost sterile fluid, but this dogma has been revised over the years. Studies in primates have reported that rhesus monkey (Macaca 
mulatta) milk contains culturable strains of at least 19 species of bacteria belonging to at least five different genera [32, 33]. Similar results have been described in other mammals [34].

Traditionally, the presence of microbes in human milk has been confirmed by use of culturedependent techniques. Most of the bacteria isolated from milk belong to Staphylococcus, Streptococcus, and Lactobacillus and Bifidobacterium spp., latter two genera having several strains with a long record of use as probiotics. [35]. It has been stated that "human milk constitutes one of the main sources of bacteria to the breastfed infant gut since an infant consuming approximately $800 \mathrm{~mL} /$ day of milk would ingest between $1 \times 10^{5}$ and $1 \times 10^{7}$ bacteria daily" $[26,27]$. Moreover, the biological role of these microbes on neonatal health at short and long-term has not yet been identified.

The origin of breast milk bacteria is currently not known, but their presence corresponds to a perinatal period which starts during the third trimester of pregnancy and continues through lactation [25]. Several reports have proposed that the human milk microbiota could derive from colonization from the mother's skin, the infant's oral cavity during suckling, or the mother's gut via the entero-mammary pathway $[1,26,27,36]$. However, a commensal microbiota has been reported in human breast tissue $[37,38]$, suggesting that specific microbes inhabit the breast tissue and potentially colonize the milk ducts.

With the development and application of culture-independent techniques (most of them based on PCR techniques) and next-generation sequencing platforms, the presence of microbial DNA has been confirmed. These techniques have confirmed the existence of a rich and diverse breast milk microbial community [20- 27, 35].

Human milk harbors a unique microbial ecosystem which differs from any other in humans. The milk microbiota is not linked with any mucosal or fecal samples, nor does it seem to be a subset of any other specific human sample [21]. The breast milk microbial diversity has been evaluated by several independent research groups [20-27, 35]. These researches have shown that Staphylococcus spp., Streptococcus spp., are the common groups followed by specific lactic acid bacteria. The first study on the milk microbiome using pyrosequencing demonstrated that 
milk bacterial communities are generally complex [20]. It was reported a high inter-individual variation regarding the number and abundance of different species in human milk, however, a common core of nine bacterial groups were present in the milk samples of each individual (Streptococcus, Staphylococcus, Serratia, Pseudomonas, Corynebacterium, Ralstonia, Propionibacterium, Sphingomonas, and Bradyrhizobiaceae) [20]. Difference results between studies may be due to different sampling and process protocols and varied DNA extraction, selection of specific primers with high bacterial coverage including bacteria with higher content of $\mathrm{G}+\mathrm{C}$ as Bifidobacterium spp and sequencing platforms; thus, a more standardized approach is needed in the future $[20,21,23,35,39]$.

The isolation and detection of live bacteria and the presence of strictly anaerobic species, such as Bifidobacterium, Clostridium, and Bacteroides [23, 25] usually associated with gut environments and unable to survive in aerobic locations, have resulted in a scientific debate on the origin of milk-associated bacteria. Recently, high-throughput sequencing has indicated the presence of gut-associated, strictly anaerobic microbes belonging to the Clostridia family (Blautia, Clostridium, Collinsella, and Veillonella) in breast milk. It has been reported the presence of specific microbes shared between the maternal microbiota, breast milk, and infant intestinal microbiota $[24,28-31]$ and also, in other maternal-neonatal niches as meconium, placenta and amniotic fluid [40]. Furthermore, the presence of butyrate-producing bacteria, including Coprococcus, Faecalibacterium, and Roseburia, has been confirmed and shown to be shared in both maternal feces and human milk.

Shotgun metagenomics analysis of human milk by total DNA sequencing using Illumina nextgeneration sequencing technology has been done using 10 pooled milk samples [41]. This study reported that human milk contains over 360 prokaryotic genera, with Proteobacteria (65\%) and Firmicutes (34\%) as the predominant phyla, and with Pseudomonas (61.1\%), Staphylococcus (33.4\%), and Streptococcus (0.5\%) as the predominant genera [41]. This study also showed that the most abundant open reading frames (ORFs) within human milk encoded proteins for basic cellular functions (respiration, cell signaling, RNA, DNA, and amino acid metabolism). 
1 A recent study [42] has reported on the metagenome and microbiota of healthy human milk

2 ( $\mathrm{n}=10$ mothers) and compared it with the milk metagenome of women suffering from mastitis.

3 A healthy core microbiome included Staphylococcus, Streptococcus, Bacteroides,

4 Faecalibacterium, Ruminococcus, Lactobacillus, and Propionibacterium, as well as fungal,

5 protozoa-related, and viral-related sequences, while the milk microbiota of women with mastitis

$6 \quad$ was dominated by Staphylococcus aureus [42].

7 Molecular techniques have some limitations. For example, the viability of milk microbes cannot

8 be analyzed, and total bacteria counts may be over- or underestimated because of cell-wall

9 composition, DNA extraction methods, and the number of microbial 16S gene copies which possible lead the over- or underestimation of bacteria counts. The DNA contamination in extraction kits and reagents has also been reported [35]. Other potential factors influencing milk microbiota composition include sampling methods (e.g., aseptic methods, time of day, breast cleaning methods, sampling before or after suckling, manual extraction or breast pump extraction), DNA extraction methods (e.g., from whole or defatted milk, use of commercial kits, enzymatic lysis, bead-beating step), sequencing platforms (Illumina, Solid, Ion Torrent, 454 Roche), 16S bacterial gene region studies (V1-V3; V3-V4, V4, etc.), and the 16S database and bioinformatics pipeline used. Thus, there is a need to standardize protocols and validate methods.

\section{Potential factors influencing the milk microbiome}

It is known that genetic factors, mode of delivery, maternal nutrition, time of day, lactation stage, and geographical region influence human milk composition, and considerable interindividual variation has also been reported [43]. Similarly, all the factors that could modulate the microbiota of the mother's skin, oral cavity, vagina, and intestine and the microbiota of the infant are potentially able to modulate the human milk microbiota. Therefore, the lactation period, maternal dietary habits and nutritional status, mode of delivery, gestational age, geographical location, and the use of antibiotics or other medicines can all have an influence on the milk microbiota. 
1 Higher microbial diversity has been reported in colostrum samples than in mature milk.

2 Lactation stage has been described as a factor influencing milk microbes [20, 21]. Initially, the

3 microbiota is dominated by Weissella, Leuconostoc, Staphylococcus, Streptococcus, and

4 Lactococcus spp. [21]. Later, the microbiota contains high levels of Veillonella, Prevotella,

5 Leptotrichia, Lactobacillus, Streptococcus, and increasing levels of Bifidobacterium and

$6 \quad$ Enterococcus spp. $[21,44]$.

7 The mode of delivery affects human milk microbiota composition. High microbial diversity and

8 high prevalence of Bifidobacterium and Lactobacillus spp. are found in colostrum and milk

9 following vaginal delivery, while the contrary is observed following caesarean delivery [21, 22, 44-46] although other studies did not report differences in microbial profiles based on gestation, mode of delivery or infant gender [47]. Breast milk microbiota composition is also influenced by gestational age, with significant differences between term- and preterm-delivered mothers. Lower counts of Enterococcus in colostrum and higher counts of Bifidobacterium in milk have been detected in samples from mothers with term deliveries [44]. In addition, changes in the milk microbiota composition are associated with maternal physiological status, including obesity, celiac disease, and HIV $[21,48-50]$. Obesity is reflected in the levels of Bifidobacterium spp and cytokines in human milk [50], as well as increased Staphylococcus spp., leptin, and pro-inflammatory fatty acid levels [50-53] and reduced microbial diversity [21]. Mothers with celiac disease have reduced levels of cytokines, Bacteroides spp. and Bifidobacterium spp. in their milk [48]. Finally, the milk from HIV-positive women from Africa has been found to show higher bacterial diversity and higher prevalence of Lactobacillus spp. than the milk of non-HIV women [49]. Analysis of the human milk microbiota shows that, in general, Staphylococcus and Streptococcus genus and also, lactic acid bacteria strains are present in milk [19- 24], but their relative amounts and the presence of other bacteria, could be dependent on geographical location [37, 49]. However, large-scale studies with breast milk samples from different geographic locations are needed. It is also evident that perinatal use of antibiotics has an impact on the maternal microbiota, including HM microbiota, affecting the prevalence of 
1 Lactobacillus, Bifidobacterium, and Staphylococcus spp. [45,54] and decreasing the abundance

2 of Bifidobacterium, Staphylococcus, and Eubacterium spp. in milk samples [37]. Chemotherapy

3 has also been associated with alteration in the milk microbiome and with a reduction in bacterial

4 diversity [55]. Studies on the impact of maternal diet on milk microbiota are few in number, but

5 it is likely that nutritional habits, which are able to modulate intestinal microbiota and human

6 milk nutritional composition, may exert changes in the milk microbiota. For example, shared

7 microbial features have been reported between bacteria present in local foods and other-

8 fermented foods and mother-infant microbial gut and breast milk [56].

9 Moreover, diet clearly influences the lipid profile of human milk, modulating the concentration of long-chain polyunsaturated fatty acids and the ratio of $\omega-3$ to $\omega-6[57,58]$. These fatty acids have immunomodulatory properties in nursing children [59], and it has recently been demonstrated in animal models that they are able to modulate gut microbiota composition in early life [60]. The consumption of probiotics and prebiotics during pregnancy could influence the human milk microbiota, but more studies are necessary to document the potential transfer of gut bacteria to the mammary glands and the impact of specific strains of bacteria [61]. On the other hand, it has been reported that nonnutritive sweeteners, such as saccharin, sucralose, and acesulfame potassium, were present in $65 \%$ of breast milk samples analyzed [62]. Those data suggest that maternal diet could modulate the bioactive compounds and microbes present in breast milk. Studies are urgently needed to investigate interactions among nutrients in the maternal diet and the breast milk microbiota and their health effects on infants.

\section{Potential strategies to modulate the maternal breast milk microbiota}

As mentioned above, diet is likely a powerful tool to alter gut microbiota. Dietary strategies, then, could be devised to modulate the microbial composition in order to affect human physiology and reduce the risk of diseases related to imbalances of microbiota composition. A recent study [63], with Japanese macaques (Macaca fuscata) as animal model, has reported the impact of maternal diet on neonatal microbiome composition. This study showed the effect of maternal high fat diet affects infant microbiome composition and activity and also, affects the 
metabolic health. Thus, deciphering the contribution of specific gut bacteria and promoting nutrition and lifestyle counseling may open new tools to reduce the risk of diseases related to microbial composition shifts. Accumulating evidence shows that early dietary interventions and nutrition counselling would support health programming effects (immunological, metabolic and microbial effect) on adult health.

It has been demonstrated that specific probiotic strains are effective in the prevention and treatment of infectious diseases in early life [64] and also, reducing the risk of eczema in infants in at-risk populations [65]. In addition, a specific probiotic bacterium, L. reuteri, has been detected in breast milk and infant feces from mothers consuming this probiotic [66]. In a placebo-controlled study, probiotics ingestion during pregnancy and breastfeeding period has been describe to modulate in infant Bifidobacteria colonization and also to modulate breast milk microbiome $[67,68]$. Recently, it has been suggested that perinatal probiotic supplementation affects breast milk composition in terms of microbes, including Bifidobacterium and Lactobacillus spp., and also affects other bioactive compounds as human milk oligosaccharides and lactoferrin [69]. Moreover, the same study found only beneficial effects of probiotic supplementation in vaginal birth, while no significant differences were found in milk samples from C-section deliveries, suggesting probiotic- specific dependent modulation depending on mode of delivery. A recent workshop report on the use of specific probiotics during first 1500 days of life supports healthy perinatal life with lowered risk of infections and autoimmune problems later in life [70].

\section{Conclusions}

Several perinatal factors influence microbial transfer from mother to infant via breast milk. The lactation period may provide a new target for devising novel dietary and nutritional tools to modulate the milk microbiota and thereby reduce the risk of noncommunicable diseases (NCDs), while at the same time promoting breastfeeding. To continue to increase our knowledge of the milk microbiota, further studies from different geographical regions and among varying population groups with different genetic backgrounds and environmental and 
1 nutrition conditions are required to fully understand the impact of the microbiome and its

2 potential in infant health promotion.

3

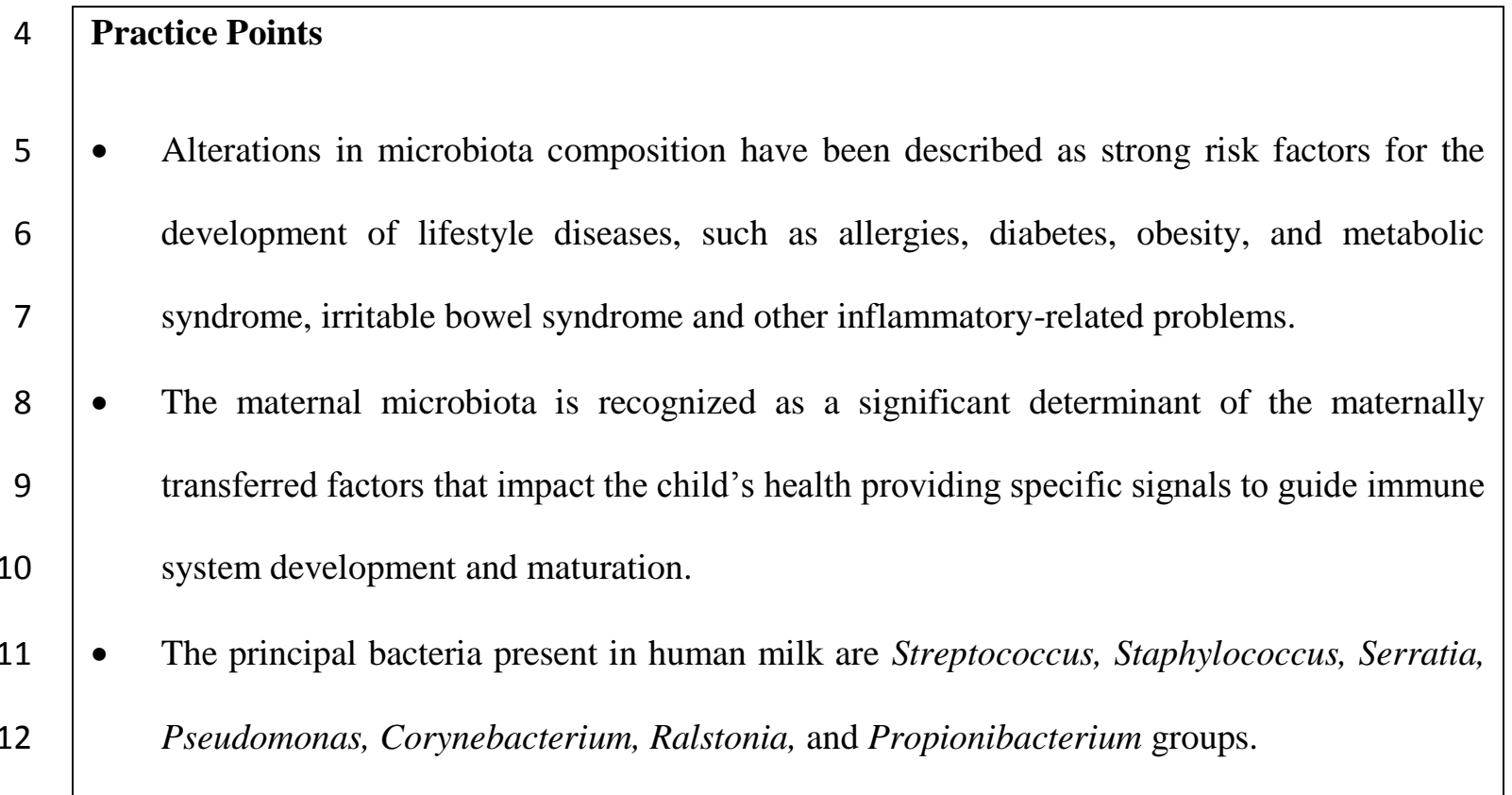

\section{Research}

- The origin of the bacteria in breast milk (colonization from the mother's skin, the infant's oral cavity during suckling, or the mother's gut).

- Clarification of the potential impact of each different species of milk bacteria on infant health.

- A Standardized protocols and methodology to analyze milk microbiota.

- Influence of the geographical location, genetic background and diet in milk microbiota.

- Use of prebiotics, probiotics and symbiotics to modulate breast milk microbiota and improve infant health. 
2 None declared.

3

4

5

6

\section{Funding sources} Murcia).

\section{References} 13(5):487-9. 156(2): S3-7

\section{Conflict of interest statement}

This review has been written within the European Research Council starting grant, MAMI project under grant agreement No. 639226. M.C. Collado is involved in the "ISCH COST Action IS1405" entitled Building Intrapartum Research Through Health e an interdisciplinary whole system approach to understanding and contextualising physiological labour and birth (BIRTH). C. Gomez- Gallego is a recipient of the Seneca Postdoctoral Grant from the Seneca Foundation, the Regional Agency of Science and Technology of the Region of Murcia (funded by the Education and Universities Council e Autonomous Community of the Region of

[1] Rautava S, Luoto R, Salminen S, Isolauri E. Microbial contact during pregnancy, intestinal colonization and human disease. Nat Rev Gastroenterol Hepatol 2012; 9(10):565-76.

[2] Bendiks M, Kopp MV. The relationship between advances in understanding the microbiome and the maturing hygiene hypothesis. Curr Allergy Asthma Rep 2013;

[3] Hooper LV, Littman DR, Macpherson AJ. Interactions between the microbiota and the immune system. Science 2012; 336: 1268-73.

[4] Marchesi JR, Adams DH, Fava F, Hermes GD, Hirschfield GM, Hold G, et al. The gut microbiota and host health: a new clinical frontier. Gut 2016; 65(2):330-9.

[5] Dunlop AL, Mulle JG, Ferranti EP, Edwards S, Dunn AB, Corwin EJ. Maternal Microbiome and Pregnancy Outcomes That Impact Infant Health: A Review. Adv Neonatal Care 2015;15(6):377-85

[6] Gomez de Agüero M, Ganal-Vonarburg SC, Fuhrer T, Rupp S, Uchimura Y, Li H, et al. The maternal microbiota drives early postnatal innate immune development. Science 2016; 351(6279):1296-302.

[7] Rodríguez JM, Murphy K, Stanton C, Ross RP, Kober OI, Juge N, et al. The composition of the gut microbiota throughout life, with an emphasis on early life. Microb Ecol Health Dis 2015 ; 26: 26050.

[8] Hooper LV, Macpherson AJ. Immune adaptations that maintain homeostasis with the intestinal microbiota. Nat Rev Immunol 2010; 10: 159-69.

[9] Walker A. 2010. Breast milk as the gold standard for protective nutrients. J Pediatr 2010; 
[10] Aaltonen J, Ojala T, Laitinen K, Poussa T, Ozanne S, Isolauri E.Impact of maternal diet during pregnancy and breastfeeding on infant metabolic programming: a prospective randomized controlled study. Eur J Clin Nutr 2011; 65(1): 10-9.

[11] Le Huërou-Luron I, Blat S, Boudry, G. 2010. Breast- v. formula-feeding: impacts on the digestive tract and immediate and long-term health effects. Nutr Res Rev 2010; 23(1): 2336.

[12] Turfkruyer M, Verhasselt V. Breast milk and its impact on maturation of the neonatal immune system. Curr Opin Infect Dis 2015; 28(3):199-206.

[13] Guaraldi F, Salvatori G. Effect of breast and formula feeding on gut microbiota shaping in newborns. Front Cell Infect Microbiol 2012;2:94.

[14] Vallés Y, Artacho A, Pascual-García A, Ferrús ML, Gosalbes MJ, Abellán JJ, et al. Microbial succession in the gut: directional trends of taxonomic and functional change in a birth cohort of Spanish infants. PLoS Genetics 2014; 10(6): e1004406.

[15] Roger LC, Costabile A, Holland DT, Hoyles L, McCartney AL. Examination of faecal Bifidobacterium populations in breast- and formula-fed infants during the first 18 months of life. Microbiology 2010; 156(11): 3329-41.

[16] Azad MB, Konya T, Maughan H, Guttman DS, Field CJ, Chari RS, et al. Gut microbiota of healthy Canadian infants: profiles by mode of delivery and infant diet at 4 months. CMAJ 2013;185(5):385-94.

[17] O'Sullivan A, Farver M, Smilowitz JT. The Influence of Early Infant-Feeding Practices on the Intestinal Microbiome and Body Composition in Infants. Nutr Metab Insights 2015;8(1):1-9.

[18] Madan JC, Hoen AG, Lundgren SN, Farzan SF, Cottingham KL, Morrison HG, et al. Association of Cesarean Delivery and Formula Supplementation With the Intestinal Microbiome of 6-Week-Old Infants. JAMA Pediatr 2016;170(3):212-9.

[19] Martin R, Langa S, Reviriego C, Jimenez E, Marín ML, Xaus J, et al. Human milk is a source of lactic acid bacteria for the infant gut. J Pediatr $2003 ; 143: 754-758$.

[20] Hunt KM, Foster JA, Forney LJ, Schütte UM, Beck DL, Abdo Z, et al. Characterization of the diversity and temporal stability of bacterial communities in human milk. PLoS One 2011; 6:e21313.

[21] Cabrera-Rubio R, Collado MC, Laitinen K, Salminen S, Isolauri E, Mira A. The human milk microbiome changes over lactation and is shaped by maternal weight and mode of delivery. Am J Clin Nutr 2012; 96(3):544-51.

[22] Cabrera-Rubio R, Mira-Pascual L, Mira A, Collado MC. Impact of mode of delivery on the milk microbiota composition of healthy women. J Dev Orig Health Dis 2016;7(1):5460. 
[23] Jost T, Lacroix C, Braegger C, Chassard C. Assessment of bacterial diversity in breast milk using culture-dependent and culture-independent approaches. Br J Nutr 2013; 110(7) : 1253-1262.

[24] Jost T, Lacroix C, Braegger C, Rochat F, Chassard C. Vertical mother-neonate transfer of maternal gut bacteria via breastfeeding. Environ Microbiol 2014; 16(9): 2891-2904.

[25] Jost T, Lacroix C, Braegger C, Chassard C. Impact of human milk bacteria and oligosaccharides on neonatal gut microbiota establishment and gut health. Nutr Rev 2015; 73(7): 426-437.

[26] Fernández L, Langa S, Martín V, Maldonado A, Jiménez E, Martín R, et al. The human milk microbiota: origin and potential roles in health and disease. Pharmacol Res 2013; 69(1): $1-10$.

[27] Jeurink PV, van Bergenhenegouwen J, Jiménez E, Knippels LM, Fernández L, Garssen J, et al. Human milk: a source of more life than we imagine. Benef Microbes 2013;4(1):1730 .

[28] Martín V, Maldonado-Barragán A, Moles L, Rodriguez-Baños M, Campo RD, Fernández L, et al. Sharing of bacterial strains between breast milk and infant feces. J Hum Lact 2012;28(1):36-44.

[29] Makino H, Kushiro A, Ishikawa E, Kubota H, Gawad A, Sakai T, et al. Mother-to-infant transmission of intestinal bifidobacterial strains has an impact on the early development of vaginally delivered infant's microbiota. PLoS One 2013; 8(11): e7833.

[30] Makino H, Martin R, Ishikawa E, Gawad A, Kubota H, Sakai T, et al. Multilocus sequence typing of bifidobacterial strains from infant's faeces and human milk: are bifidobacteria being sustainably shared during breastfeeding? Benef Microbes 2015; 6(4):563-72.

[31] Benito D, Lozano C, Jiménez E, Albújar M, Gómez A, Rodríguez JM, et al. Characterization of Staphylococcus aureus strains isolated from faeces of healthy neonates and potential mother-to-infant microbial transmission through breastfeeding. FEMS Microbiol Ecol 2015; 91(3): pii: fiv007.

[32] Jin L, Hinde K, Tao L. Species diversity and relative abundance of lactic acid bacteria in the milk of rhesus monkeys (Macaca mulatta). J Med Primatol 2011;40(1):52-8.

[33] O'Sullivan A, He X, McNiven EM, Hinde K, Haggarty NW, Lönnerdal B, et al. Metabolomic phenotyping validates the infant rhesus monkey as a model of human infant metabolism. J Pediatr Gastroenterol Nutr 2013;56(4):355-63.

[34] Quigley L, O'Sullivan O, Stanton C, Beresford TP, Ross RP, Fitzgerald GF, et al. The complex microbiota of raw milk. FEMS Microbiol Rev 2013; 37(5): 664-98.

[35] McGuire MK, McGuire MA. Human milk: mother nature's prototypical probiotic food? Adv Nutr 2015; 6(1): 112-123. 
[36] Rodríguez JM. The origin of human milk bacteria: is there a bacterial entero-mammary pathway during late pregnancy and lactation? Adv Nutr 2014; 5(6): 779-784.

[37] Urbaniak C, Cummins J, Brackstone M, Macklaim JM, Gloor GB, Baban CK, et al. Microbiota of human breast tissue. Appl Environ Microbiol 2014; 80(10):3007-14.

[38] Xuan C, Shamonki JM, Chung A, Dinome ML, Chung M, Sieling PA, et al. Microbial dysbiosis is associated with human breast cancer. PLoS One 2014; 9(1): e83744.

[39] Sim K, Cox MJ, Wopereis H, Martin R, Knol J, Li MS, et al. Improved detection of bifidobacteria with optimised $16 \mathrm{~S}$ rRNA-gene based pyrosequencing. PLoS One 2012;7(3):e32543.

[40] Collado MC, Rautava S, Aakko J, Isolauri E, Salminen S. 2016. Human gut colonisation may be initiated in utero by distinct microbial communities in the placenta and amniotic fluid. Scientific Report 2016; in press

[41] Ward TL, Hosid S, Ioshikhes I, Altosaar I. Human milk metagenome: a functional capacity analysis. BMC Microbiol 2013; 13:116.

[42] Jiménez E, de Andrés J, Manrique M, Pareja-Tobes P, Tobes R, Martínez-Blanch JF, et al. Metagenomic Analysis of Milk of Healthy and Mastitis-Suffering Women. J Hum Lact 2015; 31(3):406-15.

[43] Quinn EA, Largado F, Power M, Kuzawa CW. Predictors of breast milk macronutrient composition in Filipino mothers. Am J Human Biol 2012; 24(4), 533-540.

[44] Khodayar-Pardo P, Mira-Pascual L, Collado MC, Martínez-Costa C. Impact of lactation stage, gestational age and mode of delivery on breast milk microbiota. J Perinatol 2014; 34, 599-605.

[45] Soto A, Martín V, Jiménez E, Mader I, Rodríguez JM, Fernández L. Lactobacilli and Bifidobacteria in human breast milk: influence of antibiotherapy and other host and clinical factors. J Pediatr Gastroenterol Nutr 2014;59(1):78-88.

[46] Hoashi M, Meche L, Mahal LK, Bakacs E, Nardella D, Naftolin F, et al. Human Milk Bacterial and Glycosylation Patterns Differ by Delivery Mode. Reprod Sci 2015 pii: 1933719115623645.

[47] Urbaniak C, Angelini M, Gloor GB, Reid G. Human milk microbiota profiles in relation to birthing method, gestation and infant gender. Microbiome 2016; 4(1):1

[48] Olivares M, Albrecht S, De Palma G, Ferrer MD, Castillejo G, Schols HA, Sanz Y. Human milk composition differs in healthy mothers and mothers with celiac disease. Eur J Nutr 2015;54(1):119-28

[49] González R, Maldonado A, Martín V, Mandomando I, Fumadó V, Metzner KJ, et al. Breast milk and gut microbiota in African mothers and infants from an area of high HIV prevalence. PLoS One 2013; 8:e80299. 
[50] Collado MC, Laitinen K, Salminen S, Isolauri E. Maternal weight and excessive weight gain during pregnancy modify the immunomodulatory potential of breast milk. Pediatr Res 2012; 72(1): 77-85.

[51] Panagos P, Matthan N, Sen S. Effects of maternal obesity on breastmilk composition and infant growth. The Faseb Journal 2014; 28(1):247.7

[52] Andreas NJ, Kampmann B, Mehring Le-Doare K. Human breast milk: A review on its composition and bioactivity. Early Hum Dev 2015; 91(11), 629-35.

[53] Panagos PG, Vishwanathan R, Penfield-Cyr A, Matthan NR, Shivappa N, Wirth MD, et al. Breastmilk from obese mothers has pro-inflammatory properties and decreased neuroprotective factors. J Perinatol. 2016 doi: 10.1038/jp.2015.199

[54] Witt A, Mason MJ, Burgess K, Flocke S, Zyzanski S. A case control study of bacterial species and colony count in milk of breastfeeding women with chronic pain. Breastfeed Med 2014;9(1):29-34.

[55] Urbaniak C, McMillan A, Angelini M, Gloor GB, Sumarah, M, Burton JP, et al. Effect of chemotherapy on the microbiota and metabolome of human milk, a case report. Microbiome 2014; 2:24.

[56] Albesharat R, Ehrmann MA, Korakli M, Yazaji S, Vogel RF. Phenotypic and genotypic analyses of lactic acid bacteria in local fermented food, breast milk and faeces of mothers and their babies. Syst Appl Microbiol 2011; 34(2):148-55.

[57] Nishimura RY, Barbieiri P, Castro GS, Jordão AA Jr, Perdoná Gda S, Sartorelli DS. Dietary polyunsaturated fatty acid intake during late pregnancy affects fatty acid composition of mature breast milk. Nutrition 2014; 30(6), 685-689.

[58] Peng Y, Zhou T, Wang Q, Liu P, Zhang T, Zetterström R, et al. Fatty acid composition of diet, cord blood and breast milk in Chinese mothers with different dietary habits. Prostaglandins Leukot Essent Fatty Acids 2009; 81(5-6):325-30.

[59] Hoppu U, Isolauri E, Laakso P, Matomäki J, Laitinen K. Probiotics and dietary counselling targeting maternal dietary fat intake modifies breast milk fatty acids and cytokines. Eur J Nutr. 2012;51(2):211-9.

[60] Pusceddu MM, El Aidy S, Crispie F, O'Sullivan O, Cotter P, Stanton C, et al. N-3 Polyunsaturated Fatty Acids (PUFAs) Reverse the Impact of Early-Life Stress on the Gut Microbiota. PLoS One 2015; 10(10):e0139721.

[61] Dotterud CK, Avershina E, Sekelja M, Simpson MR, Rudi K, Storrø O, et al. Does Maternal Perinatal Probiotic Supplementation Alter the Intestinal Microbiota of Mother and Child? J Pediatr Gastroenterol Nutr 2015; 61(2):200-7.

[62] Sylvetsky AC, Gardner AL, Bauman V, Blau JE, Garraffo HM, Walter PJ, et al. Nonnutritive sweeteners in breast milk. J Toxicol Environ Health A 2015; 78(16):1029-32. 
[63] Ma J, Prince AL, Bader D, Hu M, Ganu R, Baquero K, et al. High-fat maternal diet during pregnancy persistently alters the offspring microbiome in a primate model. Nat Commun 2014; 5:3889.

[64] Taipale TJ, Pienihäkkinen K, Isolauri E, Jokela JT, Söderling EM. Bifidobacterium animalis subsp. lactis BB-12 in reducing the risk of infections in early childhood. Pediatr Res. 2016 Jan;79(1-1):65-9.

[65] Rautava S, Kainonen E, Salminen S, Isolauri E. Maternal probiotic supplementation during pregnancy and breast-feeding reduces the risk of eczema in the infant. J Allergy Clin Immunol 2012;130(6):1355-60.

[66] Abrahamsson TR, Sinkiewicz G, Jakobsson T, Fredrikson M, Björkstén B. Probiotic lactobacilli in breast milk and infant stool in relation to oral intake during the first year of life. J Pediatr Gastroenterol Nutr 2009;49(3):349-54.

[67] Grönlund MM, Gueimonde M, Laitinen K, Kociubinski G, Grönroos T, Salminen S, et al. Maternal breast-milk and intestinal bifidobacteria guide the compositional development of the Bifidobacterium microbiota in infants at risk of allergic disease. Clin Exp Allergy 2007; 37(12):1764-72.

[68] Gueimonde M, Sakata S, Kalliomäki M, Isolauri E, Benno Y, Salminen S. Effect of maternal consumption of Lactobacillus GG on transfer and establishment of fecal bifidobacterial microbiota in neonates. J Pediatr Gastroenterol Nutr 2006;42(2):166-70.

[69] Mastroimarino P, Capobianco D, Miccheli A, Praticò G, Campagna G, Laforgia N, et al. Administration of a multistrain probiotic product (VSL\#3) to women in the perinatal period differentially affects breast milk beneficial microbiota in relation to mode of delivery. Pharmacol Res 2015; 95-96 : 63-70.

[70] Reid G, Kumar H, Khan AI, Rautava S, Tobin J, Salminen S. The case in favour of probiotics before, during and after pregnancy: insights from the first 1,500 days. Benef Microbes 2016; 3:1-10. 